\title{
Perineural Spread of Distant Squamous Cell Carcinoma to the Orbit: A Case Report and Review
}

\author{
Bina Gurung ${ }^{1}$, Michael Kazim, ${ }^{*}$, Gary J. Lelli, Jr. ${ }^{3}$ and Joseph Walrath ${ }^{4}$ \\ ${ }^{I}$ Faculty of Medicine, Edward Ford Building, University of Sydney, Camperdown, NSW 2000 Australia \\ ${ }^{2}$ Harkness Eye Institute, Columbia Presbyterian Hospital, 635 W. 165 Street, NY 10032, New York \\ ${ }^{3}$ Weill Cornell Department of Ophthalmology, Division of Oculoplastic Surgery, New York Presbyterian Hospital, 1305 \\ York Avenue, NY 10021, New York \\ ${ }^{4}$ Harkness Eye Institute, Columbia Presbyterian Hospital, 635 W. 165 Street, NY 10032, New York
}

\begin{abstract}
An 80 year-old man presented subacutely with a well-circumscribed superior orbital mass and associated ipsilateral numbness in the frontal nerve distribution. Histopathology after open biopsy revealed well-differentiated invasive squamous cell carcinoma [SCC]. Though no previous history of cancer was elicited from the patient, his dermatologist reported removing three squamous cell carcinomas from the right forehead in the past ten years. Herein, we report a case of perineural orbital spread of distant primary cutaneous SCC.
\end{abstract}

Keywords: Ipsilateral numbness, perineural spread [PNS], primary cutaneous SCC, squamous cell carcinoma [SCC].

\section{INTRODUCTION}

PNS of cutaneous SCC of the head and neck, as opposed to mucosal SCC, has a relatively low incidence. Nonetheless PNS of cutaneous SCC portends a poor prognosis. A vigilant approach is warranted when a patient presents with numbness and pain. We present a case of PNS of distant primary cutaneous SCC to the orbit.

\section{CASE REPORT}

An 80 year-old man with a history of polymyalgia rheumatica and glaucoma presented with four months of a progressive sense of pressure in the right eye socket and associated bulging. Pain and numbness involved the right forehead. He noted binocular diplopia, as well as diminished vision in the right eye during the eight weeks prior to oculoplastic consultation. The patient denied any history of systemic malignancy, though he did recall having multiple skin lesions "burned off" over the past 10 years. Ophthalmic examination revealed a visual acuity of 20/40 in each eye. The right eye was injected and chemotic with five millimeters of proptosis. The pupil examination was normal. The right eye had severely limited motility in upgaze, as well as moderate limitations in downgaze and horizontal gazes. Motility of the left eye was normal. Hypoesthesia was present in the right V1 distribution, but not in the V2 or V3 distributions. Dilated funduscopic examination was normal. The Humphrey visual field test on the right eye was equivocal; the field of the contralateral eye was normal.

*Address correspondence to this author at the Harkness Eye Institute, Columbia Presbyterian Hospital, 635 W. 165 Street, NY 10032, New York; Tel: 212.305.5477; Fax: 212.923.0075; E-mail: mk48@columbia.edu
Magnetic Resonance Imaging [MRI] revealed a well circumscribed, $4.5 \times 2 \mathrm{~cm}$ cystic appearing mass extending along the right orbital roof towards the orbital apex (Fig. 1). This lesion demonstrated an irregular margin of enhancement with central necrosis or fluid suggested by the nonenhancing central portion of the lesion.

A diagnostic procedure was performed. Right lateral orbitotomy revealed a tumor that was intimately involved with the frontal nerve, and which contained a straw-colored fluid. Intraoperative histopathological evaluation of the subtotal resection returned a diagnosis of SCC. Permanent histopathologic examination revealed an invasive, moderately-differentiated keratinizing SCC within the perineurium. Focal necrosis was present. The nerve bundles themselves were not infiltrated.

The patient was treated with external beam radiation to the orbit and along the path of the frontal nerve back to the Gasserian ganglion. Exenteration was not performed, as imaging studies revealed that the disease extended to the orbital apex and it was felt that a tumor-free posterior surgical margin could not be obtained. The patient was also referred to his dermatologist for complete skin evaluation, with particular attention to the regions in which his prior SCC had been excised.

\section{DISCUSSION}

While pressure on sensory nerves from benign tumors may cause pain, numbness is often a more ominous sign. Numbness and paresthesia such as formication often suggest that a lesion is infiltrative rather than comprehensive in nature. The key symptom of numbness in the V1 distribution 


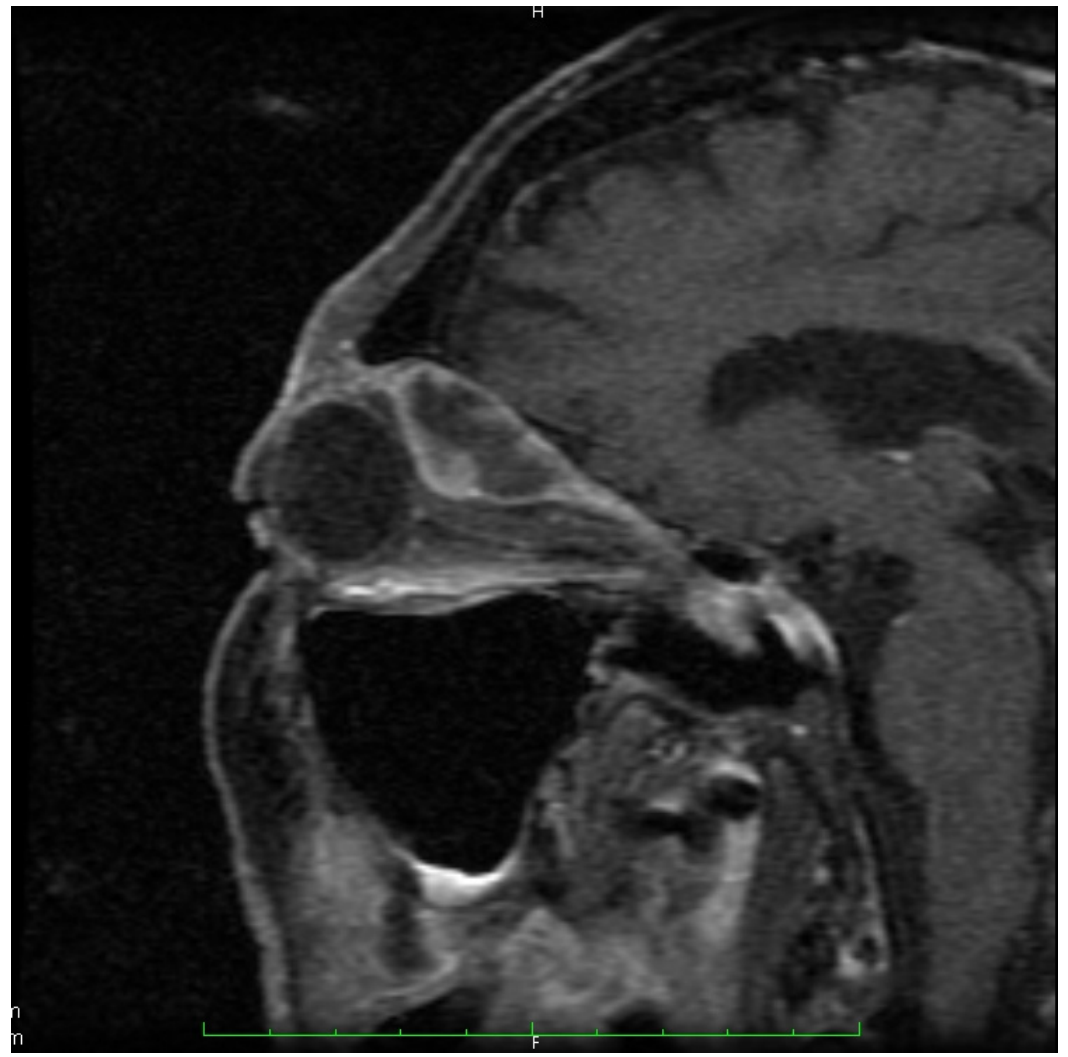

Fig. (1). A well circumscribed cystic appearing mass extending along the right orbital roof towards the orbital apex.

along with the patient's prior history of ipsilateral SCC of skin, are consistent with Morris and Joffe's description of PNS of cutaneous SCC. The initial spread involves the distal branches of the trigeminal nerve and usually progress to the larger, more proximal branches, involving larger sensory territories. Spread may also jump to the motor nerves [1]. The symptoms and signs occur indolently in some and fulminantly in others $[1,2]$.

PNS is an important characteristic of malignancies arising in the skin of head and neck, first reported by Cruveilhier in 1835 [3]. The tumor travels in the perineural space, a tissue plane of low resistance often for great distances. There is little tendency to infiltrate the actual nerve bundles - the segmental paresthesias are caused by local compression from the tumor within the perineural space, causing local infarction of the nerve [4]. These features were identified in our histopathological sections. In 239 patients with mucosal squamous carcinomas of the head and neck, PNS was demonstrated in resections of 64 patients $(27 \%)$, the majority having primary tumors at one of three sites: buccal cavity, larynx, and pharynx [5].

PNS can occur even in the setting of small isolated primary cutaneous SCC $[4,6]$. It is the rule that the primary lesion is usually treated, and there is no superficial sign of

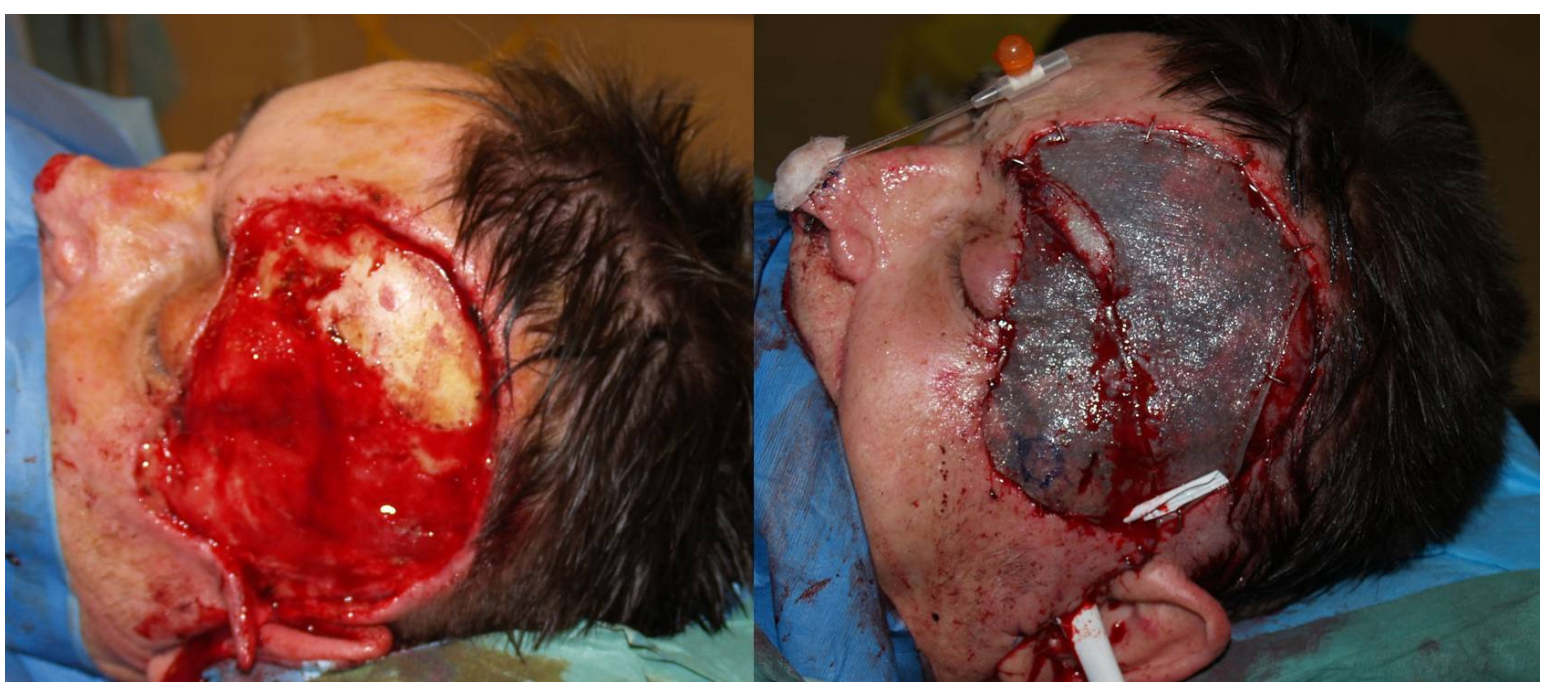

Fig. (2). Microvascular contralateral temporal fascia flap with eyebrow to reconstruct defect from a resection of a supraorbital SCC [9]. 
recurrent active disease in these patients [1]. Primary risk factors for invasive SCC include sun exposure, increased age (above 75 years), psoriasis, lymphoma and other immunodeficiency states [7]. The most common result of orbital imaging early in this disorder is that of a normal MRI of the orbit. Our case is a common orbital finding in more advanced disease: a cystic lesion centered on the trunk of sensory nerve [2]. We considered in our differential diagnosis other tumors that might present as well-circumscribed, cystic appearing, and extraconal: necrotic schwannoma, lymphangioma, and metastasis. Other malignant tumors include adenocystic carcinoma, lymphoma, and metastatic tumor [8].

Posch et al. [9] suggests treatment comprising of mapping tumor with multiple biopsies, imaging with MRI, followed by resection of the tumor. Once histopathology confirmation of the resection is obtained, a flap is reconstructed which include micro vascular fasciocutaneous flaps (Fig. 2). Our patient underwent irradiation for the unresectable disease. Bowyer et al. [2] reported mortality in 24 percent of patients treated with irradiation after 46 months follow-up. Thirty five percent of patients were alive with evidence of disease progression. McNab et al. [10] reported on 21 patients and found 46 percent and 62 percent two and three year mortality, respectively.

\section{SUMMARY}

Perineural spread of cutaneous SCC of the head and neck, as opposed to mucosal SCC, has a relatively low incidence. Nonetheless PNS of cutaneous SCC portends a poor prognosis [10]. A vigilant approach is warranted when a patient presents with numbness and pain. Diagnosis is often achieved on the basis of thorough history, examination and imaging. Biopsy is almost always indicated. All parameters are essential in potentially minimizing morbidity and mortality from PNS.

\section{ABBREVIATIONS}

$\begin{array}{lll}\text { MRI } & \text { Magnetic Resonance Imaging } \\ \text { PNS } & =\text { Perineural Spread } \\ \text { SCC } & \text { Squamous Cell Carcinoma }\end{array}$

\section{REFERENCES}

[1] Morris JG, Joffe R. Perineural spread of cutaneous basal and squamous cell carcinomas. The clinical appearance of spread into the trigeminal and facial nerves. Arch Neurol 1983; 40(7): 424-9.

[2] Bowyer JD, Sullivan TJ, Whitehead KJ, et al. The management of perineural spread of squamous cell carcinoma to the ocular adnexae. Ophthal Plast Reconstr Surg 2003; 19(4): 275-81.

[3] Cruveilhier J. Maladies des Nerfs: anatomie Pathologique du Corp Humain, $2^{\text {nd }}$ ed. Paris: Bailliere JB 1842; Part 2: 35: 3.

[4] Carter RL, Foster CS, Dinsdale EA, et al. Perineural spread by squamous carcinomas of the head and neck: a morphological study using antiaxonal and antimyelin monoclonal antibodies. J Clin Pathol 1983; 36(3): 269-75.

[5] Soo KC, Carter RL, O'Brien CJ, et al. Prognostic implications of perineural spread in squamous carcinomas of the head and neck. Laryngoscope 1986; 96(10): 1145-8.

[6] Veness MJ, Biankin S. Perineural spread leading to orbital invasion from skin cancer. Aust Radiol 2000; (44): 296-302.

[7] Mullen JT, Feng L, Xing Y, et al. Invasive squamous cell carcinoma of the skin: defining a high-risk group. Ann Surg Oncol 2006; 13(7): 902-9.

[8] Lee, Charles. Perineural spread of tumor along the fifth and seventh cranial nerves. Updated: Jun 16, 2006; Reference Available from: http://emedicine.medscape.com/article/875786-overview.

[9] Posch NA, Mureau MA, Flood SJ, Hofer SO. The combined free partial vastus lateralis with anterolateral thigh perforator flap reconstruction of extensive composite defects. Br J Plast Surg 2005: 58: 1095-103.

[10] McNab AA, Francis IC, Benger R, et al. Perineural spread of cutaneous squamous cell carcinoma via the orbit. Clinical features and outcome in 21 cases. Ophthalmology 1997; 104(9): 1457-62. 